\title{
Retraction Note to: Common polymorphism in the MMP-13 gene may contribute to the risk of human cancers: a meta-analysis
}

\author{
Ping Gao ${ }^{1}$ Jun-Lan Yang ${ }^{1} \cdot$ Hong Zhao ${ }^{2}$ Jun-Hao You $^{2} \cdot \mathrm{Yi} \mathrm{Hu}^{1}$
}

Published online: 17 August 2015

(C) International Society of Oncology and BioMarkers (ISOBM) 2015

Retraction to: Tumor Biol. (2014) 35:10137-10148

DOI 10.1007/s13277-014-2309-y

The Publisher and Editor retract this article in accordance with the recommendations of the Committee on Publication Ethics (COPE). After a thorough investigation we have strong reason to believe that the peer review process was compromised.

The online version of the original article can be found at http://dx.doi.org/ $10.1007 /$ s13277-014-2309-y.

Yi $\mathrm{Hu}$

dr_jiao301@126.com

1 Department of Oncology, Chinese PLA General Hospital, No. 28 Fuxing Road Haidian District, Beijing 100853, People's Republic of China

2 Department of Oncology, Hainan Branch of Chinese PLA General Hospital, Beijing 100853, People's Republic of China 